\title{
Developing a 3-DOF Compliant Perching Arm for a Free-Flying Robot on the International Space Station
}

\author{
In-Won Park ${ }^{1}$, Trey Smith ${ }^{2}$, Hugo Sanchez ${ }^{2}$, Sze Wun Wong ${ }^{3}$, Pedro Piacenza $^{3}$ and Matei Ciocarlie ${ }^{3}$
}

\begin{abstract}
This paper presents the design and control of the 3-DOF compliant perching arm for the free-flying Astrobee robots that will operate inside the International Space Station (ISS). The robots are intended to serve as a flexible platform for future guest scientists to use for zero-gravity robotics researchthus, the arm is designed to support manipulation research. It provides a 1-DOF underactuated tendon-driven gripper capable of enveloping a range of objects of different shapes and sizes. Co-located RGB camera and LIDAR sensors provide perception. The Astrobee robots will be capable of grasping each other in flight, to simulate orbital capture scenarios. The arm's end-effector module is swappable on-orbit, allowing guest scientists to add upgraded grippers, or even additional arm degrees of freedom. The design of the arm balances research capabilities with Astrobee's operational need to perch on ISS handrails to reduce power consumption. Basic arm functioning and grip strength were evaluated using an integrated Astrobee prototype riding on a low-friction air bearing.
\end{abstract}

\section{INTRODUCTION}

The Intelligent Robotics Group at NASA Ames Research Center is building the free-flying Astrobee robots to operate inside the International Space Station (ISS) [1]-[5]. Three Astrobee robots will operate in the ISS, and three will remain on the ground for support testing. The robots' primary function is to serve as a zero-gravity robotics research platform, replacing the free-flying SPHERES satellites that have been among the most frequently-used payloads on the ISS over the past ten years. The robots will also serve operational needs: as free-flying cameras to observe crew activities, and as platforms to carry sensors to survey the ISS interior. For example, the REALM project [6] will use an Astrobee to carry an RFID reader and take inventory of RFID-tagged items.

As a part of the Astrobee robotic system, a compliant, detachable arm is being developed. This arm will both support manipulation research and allow an Astrobee to perch on ISS handrails during long duration tasks. Perching allows the robot to minimize power consumption by idling propulsion and reducing computational load. It also keeps the robot quiet and out of the way of crew, an important advantage when it is used to capture video of crew activities.

\footnotetext{
${ }^{1}$ Author is with SGT Inc. within the Intelligent Robotics Group, NASA Ames Research Center, Moffett Field, CA 94035 USA in.w.park@nasa.gov

${ }^{2}$ Authors are with NASA Ames Research Center, Moffett Field, CA 94035 USA trey.smith@nasa.gov, hugo.sanchezenasa.gov

${ }^{3}$ Authors are with the Department of Mechanical Engineering, Columbia University, New York, NY 10027 USA pp2511@columbia.edu, matei.ciocarlie@columbia.edu
}

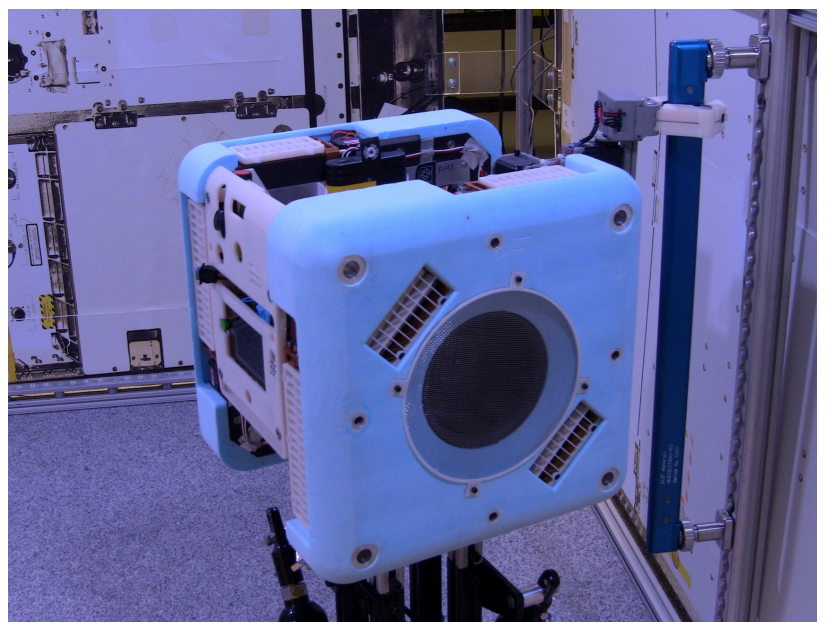

Fig. 1. A prototype of Astrobee grasping an ISS handrail on the top of micro-gravity simulating surface.

When designing the arm, we were faced with a great diversity of end effector options [7] (including suction [8]-[9], electro-adhesion [10], microspine [11]-[13], geckoadhesion [14]-[17], and underactuated grippers [18]-[22]). In order to preserve as much flexibility as possible for future researchers, we chose to: (1) make the end-effector module easily swappable on-orbit, giving researchers the option to add a new end-effector without needing to build an entirely new arm, and (2) build a baseline end-effector using mature technology that is highly reliable and can serve Astrobee's operational need for perching, while also supporting as much research as possible.

Our selected end-effector design is a 1-DOF underactuated tendon-driven gripper. We use two fingers with two revolute joints per finger, all actuated by a single tendon. Compared to a simpler and smaller parallel-jaw gripper, this type of kinematic design has the potential to perform more stable enveloping grasps on a range of objects of different sizes [21], [22]. The gripper is sized to robustly grasp ISS handrails - these handrails are attractive targets because they are located throughout the interior for crew convenience, they have uniform shape and appearance, and their rugged aluminum structure is designed to handle high loads exerted by crew (so Astrobee robots will not damage them).

In contrast, most of the exposed "walls" of the ISS interior are actually unique surfaces with different material properties, such as the front of a payload rack containing sensitive experiments, the front of a fabric bag, etc. Furthermore, much of the wall surface is cluttered with items like switches, small 
avionics boxes, and wire runs. Thus, grippers designed to adhere to flat surfaces (gecko, electro-adhesion, microspine, etc.), while highly promising, are also more complicated to use in terms of identifying open spaces to perch on, ensuring the gripper can adhere to a variety of surface materials, and obtaining permission to perch at each location.

Other advantages of the tendon-driven gripper are that it does not use high voltages (cf. electro-adhesion), does not require a bulky, vibrating vacuum pump (cf. suction, jamming), and does not require initial reaction forces that are challenging to provide given the robots' limited propulsion performance (cf. gecko, jamming).

The Astrobee arm is shown in Fig. 1. The arm's kinematic arrangement with two revolute joints is designed so that when the robot perches on a handrail, the arm can act as a pantilt unit, controlling pointing of the SciCam camera on the opposite side of the robot. The arm length is sized to avoid interference between the robot and the wall over the required pan-tilt range.

The arm is small enough to fit completely within an Astrobee payload bay when stowed. This avoids increasing the volume occupied by the robot, which is important when moving through confined spaces such as the hatchways between modules. It also keeps the stowed arm within the envelope of the robot's padded corner bumpers, which are designed to protect both the robot and the ISS from high forces in case the robot collides with ISS structure.

Part of Astrobee's safety approach is to ensure each robot is light, soft, and slow enough that it is unlikely to damage the ISS in case of a collision. This approach forces a lightweight design, making it impractical to build the arm to resist strong forces exerted by crew. We have chosen to turn this constraint into a feature by making the arm highly compliant and back-drivable. Each arm joint can detect large astronaut-induced torques in real time and deactivate automatically to allow back-driving. The 1-DOF gripper uses a torsional spring to close and an actuated tendon to open, so the gripper passively maintains its grip when unpowered, and astronauts can manually open the gripper by overcoming spring torques.

If a perched Astrobee is blocking a crew member's escape path during an emergency, they can simply push it out of the way, easily overcoming the grip strength. They can also backdrive both arm and gripper to manually perch an Astrobee, when convenient.

Note that the gripper is designed as a modular component. Crew can easily swap in a new gripper, either as a research payload or a permanent upgrade. Since the arm communication bus allows daisy chaining, a future gripper module could even provide extra arm degrees of freedom. The prototype shown in Fig. 1 has been tested with an air bearing such that the Astrobee robot is free to drift on the granite table. The prototype is able to grasp a handrail and exercise the pan motion successfully.

This paper is organized as follows. Section II describes the design of 3-DOF perching arm for Astrobee including the Astrobee payload interface, structure, and avionics. Section

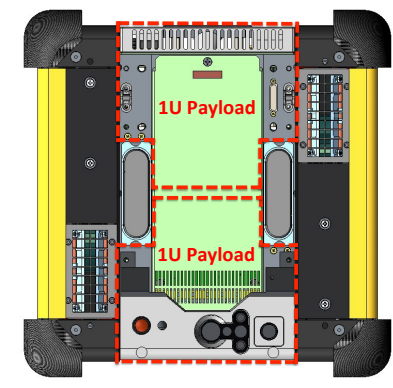

(a) Top

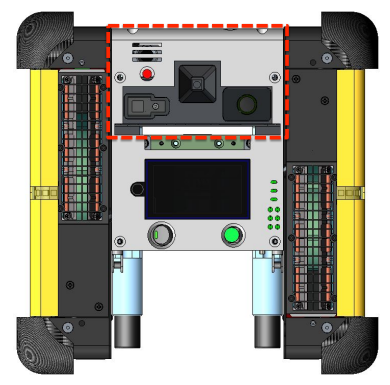

(b) Front
Fig. 2. Snapshot of the Astrobee payload bay.

III explains the process of controlling 3-DOF perching arm in real time. Section IV presents the simulation results of gripper and the experimental results of perching arm on a micro-gravity simulating surface. Concluding remarks follow in Section V.

\section{3-DOF PERCHING ARM DESIGN}

This section introduces the specification of the Astrobee payload interface, and the design for structure and avionics.

\section{A. Payload Interface}

As a research platform, the Astrobee robots must enable guest scientists to add new payloads to support their research. Astrobee provides four identical peripheral bays: top forward, top aft, bottom forward, and bottom aft (Fig. 2(a)). The top forward bay is always occupied by a collection of sensors that are critical for navigation (Fig. 2(b)). The top aft bay is normally occupied by the perching arm, but crew can easily detach the arm and replace it with a payload. Thus, three of the four bays are available for guest scientists to add new payloads that support their research. The identical layout gives payload developers the flexibility to assign any available bay to their payload on orbit, making it more feasible for multiple payloads to ride along simultaneously.

The volume available for a payload within a single bay is a box of size $123.2 \times 152.4 \times 101.6 \mathrm{~mm}(4.85 \times 6.0 \times$ $4.0 \mathrm{in}$ ); within the box there are keepouts for air flow and the robot's batteries. Electrical connectivity is provided by a 31-pin blind-mate connector (Glenair M83513-03-E03C). Each payload connector provides a nominal voltage of $14.4 \mathrm{~V}$ with a current limit of $3 \mathrm{~A}$. It has three USB 2.0 compatible pinouts (data only) that connect to different processors in the robot's core avionics [4]. Most payloads will connect to the USB bus of the high-level processor, which is dedicated to guest science. Since the arm driver is part of Astrobee's baseline flight software, it runs on the mid-level processor (MLP), and the arm connects to the MLP USB bus.

To attach a payload, a crew member slides the payload down a pair of rails until it makes contact at the bottom, then actuates two retention levers. These levers both close the electrical connection with the blind-mate connector and robustly lock the payload in place. This no-tool design allows quick payload swapping with minimal crew time. (The 


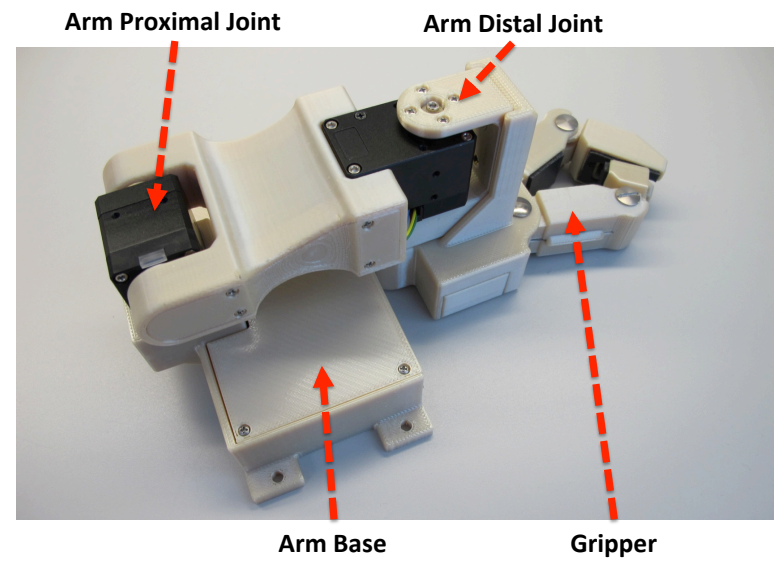

(a) Diagonal

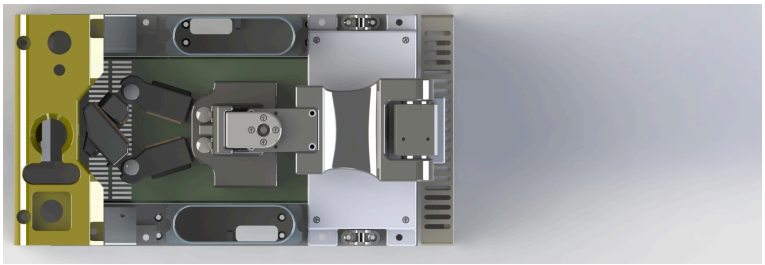

(b) Stowed Configuration

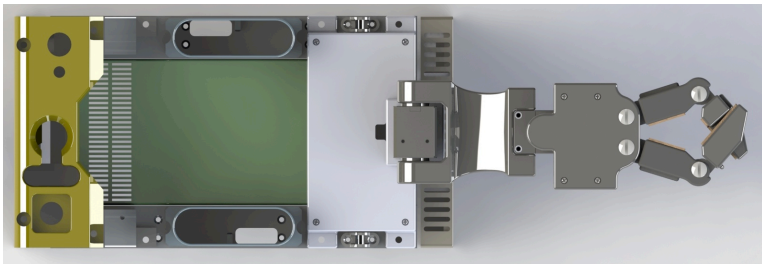

(c) Deployed Configuration

Fig. 3. Snapshot of the Astrobee perching arm.

prototype arm shown in this paper does not yet incorporate the lever mechanism.)

A "1U" payload shown in Fig. 2(a) stays completely within a single payload bay. A " $2 \mathrm{U}$ " payload occupies both of the contiguous payload bays on the bottom. By this metric, the perching arm is a " $1.5 \mathrm{U}$ " payload that stays mostly within the top aft payload bay, but also cheats into the top forward bay, while carefully avoiding interference with the top forward module, as shown in Fig. 3(b). A second identical perching arm could be mounted in either of the bottom bays, if desired.

Note that payloads could also extend outside the volume of the payload bays, but in that case they would no longer be able to rely on the robot's padded corner bumpers to protect them in a collision; they would need to develop their own collision safety plan (perhaps employing additional bumpers, or relying on crew tending for safety).

\section{B. Structure}

There are three main design drivers for Astrobee perching arm structure-size, mass, and compliance. The perching arm must stow completely inside of Astrobee payload volume so that it is not exposed to collision hazard during flight

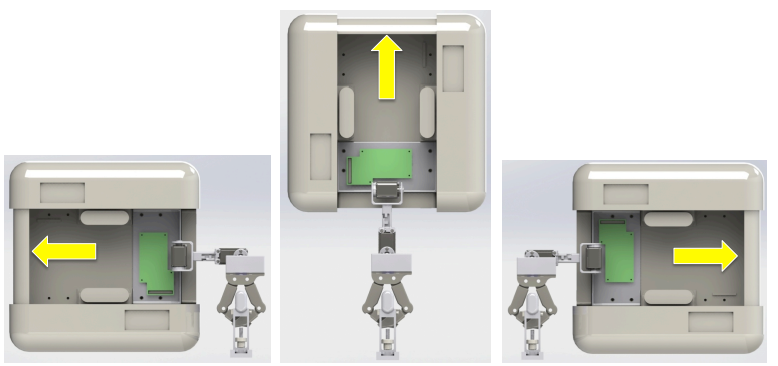

(a) Pan range of $-90.0^{\circ}$ to $90.0^{\circ}$

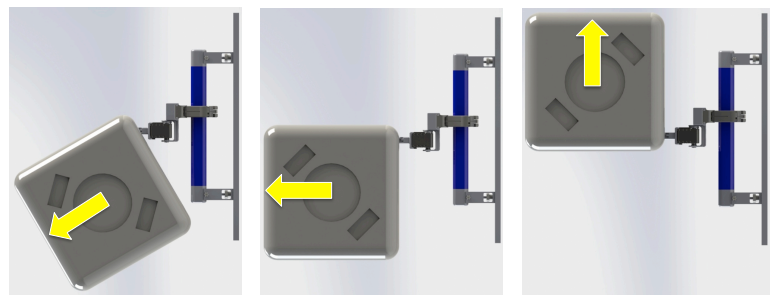

(b) Tilt range of $-30.0^{\circ}$ to $90.0^{\circ}$

Fig. 4. The pan and tilt range of Astrobee perching arm (the arrow indicates the preferred forward motion direction).

operations. The perching arm must be lightweight in order to preserve the robot's maneuverability given propulsion performance limitations (the lighter the better, target mass on the order of $500 \mathrm{~g}-1 \mathrm{~kg}$ ). The design must allow an astronaut to manually perch the arm and gripper to a handrail without requiring power from actuators. In contrast, once the actuators are powered, arm joints must be fully back-drivable and gripper must be released automatically when it detects large astronaut-induced torques.

The arm, shown in Fig. 3(a), consists of arm base, arm proximal joint, arm distal joint, and gripper. The controller board is located in the arm base. The arm proximal joint and the arm distal joint are used to stow the gripper inside of the outer structure and to operate as a pan-tilt module. The gripper is designed to grasp ISS handrails, and packaged as a replaceable modular component. All structural pieces are printed using Ultem 9085, which is the preferred 3D print material on board the ISS due to its offgassing and flammability properties.

Fig. 3(c) shows the deployed configuration. The arm deploys while in free space away from the handrail. During the perching approach, the robot transitions from generalpurpose localization (using forward-facing NavCam monocular vision) to handrail-relative localization (aft-facing PerchCam depth sensor that detects handrail geometry). Once the gripper has nominally enveloped the handrail, the robot can verify a successful grasp by reversing thrust and checking for null motion.

The perching arm is required to operate both in the zerogravity ISS environment and in $1 \mathrm{~g}$ lab testing. Thus, the arm joint torques must be specified so that the arm can support its own weight in $1 \mathrm{~g}$, but joint brakes are not needed, because they will provide little power consumption benefit when the system faces low disturbance torques on orbit. 


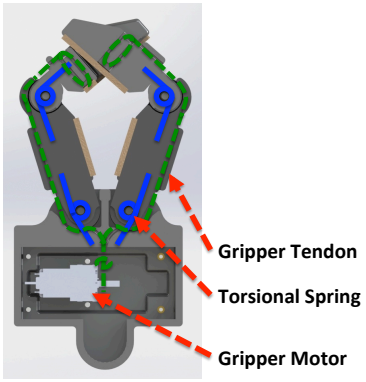

(a) Closed Configuration

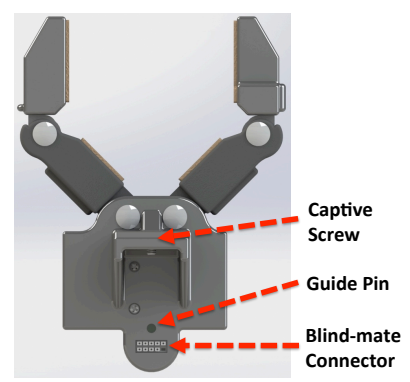

(b) Opened Configuration
Fig. 5. Snapshot of the Astrobee perching arm gripper.

The 2-DOF arm uses two Dynamixel XM430-W210 motors. Each motor has an aluminum case, which helps with heat rejection and satisfy ISS flammability requirements. The lengths of the proximal and distal links are optimized such that the 2-DOF arm stows inside the Astrobee payload volume, and provides a pan range of $-90.0^{\circ}$ to $90.0^{\circ}$ and a tilt range of $-30.0^{\circ}$ to $90.0^{\circ}$ while perched as shown in Fig. 4. The mass of the prototype arm shown in Fig. 3(a) is 733 $\mathrm{g}$ including all mechanical components, avionics, and wire harnesses; minor mass growth is expected when the finalized retention levers are integrated into the design.

We have investigated various types of gripper [7] - suction [8]-[9], electro-adhesion [10], microspine [11]-[13], geckoadhesion [14]-[17], and underactuated grippers [18]-[22]. Each type was compared using the following criteria: size, mass, compliance, grasping force, precision, and actuation time. After a trade study, suction, electro-adhesion, microspine, and gecko-adhesion gripper were rejected. The suction gripper requires a bulky vacuum pump, and the size of the state-of-art electro-adhesion gripper (Grabit 1832 gripper) is not suitable to be attached at the Astrobee perching arm. The microspine would not work on smooth surfaces like ISS handrails, and the gecko-adhesion gripper requires an additional mechanism to attach/detach and is sensitive to temperature and humidity. In the end, we selected an open source tendon-driven gripper design [23] and modified it slightly. This design offers high technical maturity, small form factor with a single actuator, compliant contact with the surface, and high grip strength through envelopment.

Fig. 5 shows the closed and opened configurations of the gripper, which consists of gripper tendons, torsional springs, and a 1-DOF gripper (Pololu DC) motor. The gripper uses torsional springs for joint flexion and an actuated tendon for extension. This allows grasping force to be maintained even with the motor turned off. It also allows external forces to open the gripper by overcoming spring torques, rather than having to back-drive the motor. Furthermore, independent flexion torques at the gripper proximal and gripper distal joints provide passive compliance to the shape of the grasped object; the perching procedure is thus robust to positioning errors with respect to the handrail. Silicone foam is attached on the inner surface of gripper to increase the contact friction
TABLE I

Blind-MATE Connector Pinout

\begin{tabular}{c|c}
\hline Pin Number & Pin Mapping \\
\hline \hline 1 & Gripper Servo Motor PWM \\
\hline 2 & Gripper DC Motor PWM- \\
\hline 3 & Gripper DC Motor PWM+ \\
\hline 4 & 5V (Maximum 1A) \\
\hline 5 & Gripper DC Encoder OUT A \\
\hline 6 & Gripper DC Encoder OUT B \\
\hline 7 & GND \\
\hline 8 & 11V (Maximum 2A) \\
\hline 9 & Arm Motor DATA+ \\
\hline 10 & Arm Motor DATA-
\end{tabular}

between ISS handrail and the gripper surface.

A total of 3 torsional springs ( 2 at the gripper proximal joint and 1 at the gripper distal joint) are used at each gripper joint to produce a grasping force. The spring coefficients of the torsional springs are maximized based on the relationship between the maximum angle of deflection for each spring and the stall torque and spool of gripper motor, where a factor of safety of 1.2 is used to account for friction and avoid over-stress on the gripper motor. When the ISS handrail is grasped as shown in Fig. 5(a), the gripping forces at the gripper proximal joint and the gripper distal joint are $3.47 \mathrm{~N}$ and $2.87 \mathrm{~N}$, respectively. When the gripper is fully opened as shown in Fig. 5(b), the gripper proximal joint makes a $45.0^{\circ}$ wide opening with respect to the gripper palm and the gripper distal link makes a $45.0^{\circ}$ wide opening with respect to the gripper proximal link, which translates to a torque of 105.9 $\mathrm{Nmm}$ and $44.3 \mathrm{Nmm}$ at the gripper proximal joint and the gripper distal joint, respectively.

The Astrobee perching arm is designed to support manipulation research. For example, it would be easy to add a grasping fixture to one robot and grasp it with the perching arm of another robot. The gripper is also designed as replaceable modular component-the existing 2-DOF arm could accommodate a new end-effector, or even a multiple-DOF "extension arm", controlled via the daisy chained RS485 bus.

As shown in Fig. 5(b), the modular gripper design provides a guide pin to assist in alignment of gripper during installation of blind-mate connector, and locks into place with two captive screws located on the perpendicular plane. Table I presents the pinout of the 10-pin blind-mate connector, which provides power and data line for one servo motor, one DC motor, and multiple arm motors.

\section{Avionics}

Fig. 6 shows the block diagram of perching arm controller board. The battery voltage (Inspired Energy ND2054) is regulated to $11 \mathrm{~V}, 5 \mathrm{~V}$, and $3.3 \mathrm{~V}$ using three regulators (2 $\times$ LM22670 and TPS62162), where the current sensors (MAX4372) are used on $11 \mathrm{~V}$ and 5V line to monitor the overall power consumption of the perching arm controller board in real time. Microprocessor dsPIC33EP512MC806 communicates with the MLP of Astrobee robot via serial bus at 115,200 Baud. Note that the open source software and 


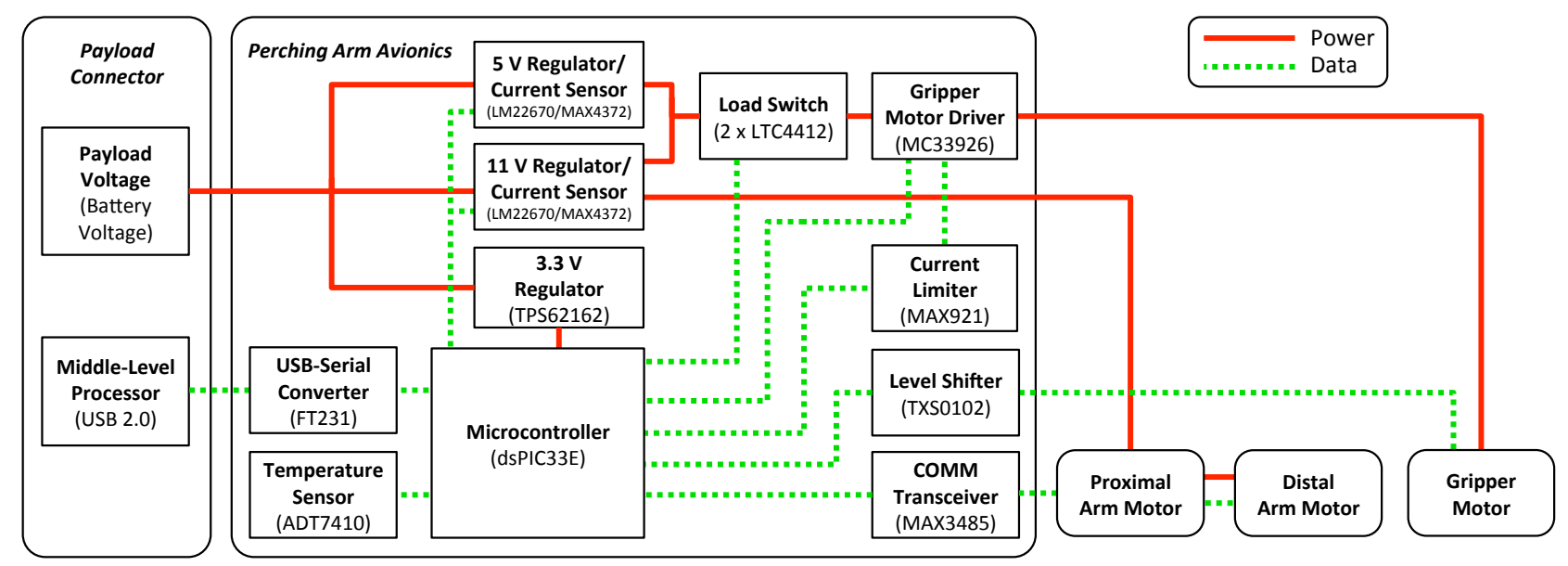

Fig. 6. Block diagram of perching arm controller board.

bootloader [24] have been modified such that the firmware of microcontroller can be remotely updated from the ground station through the MLP. During this process, the MLP resets the microcontroller by toggling the data terminal ready (DTR) line in the USB-serial converter (FT231) to allow for reprogramming the memory.

The arm motors are directly controlled from the microcontroller via RS485 protocol (MAX3485) at 115,200 Baud, and the gripper DC motor is controlled using the motor driver (MC33926). The load switch $(2 \times$ LTC4412) is used to select the gripper DC motor voltage between $11 \mathrm{~V}$ and $5 \mathrm{~V}$. A current limiter consisting of a comparator (MAX921), multivibrator (SN74LVC1G123), and flip-flop disables the motor driver when the peak current of gripper DC motor reaches $80 \%$ of the stall current. In this way, the current limiter circuit always prevents from over-temperature and over-current to guarantee the safety of the gripper DC motor. Two quadrature encoder outputs from the gripper DC motor are connected to the microcontroller, with a level shifter (TXS0102) used in between to level the voltage outputs.

\section{CONTROL}

The control algorithm of Astrobee perching arm is presented in Algorithm 1, which runs at $1000 \mathrm{~Hz}$ to receive the command from the MLP, to control 2 arm motors and 1 gripper DC motor, and to send a feedback packet to the MLP at $1 \mathrm{~Hz}$. The boolean flag control_loop_time becomes true when the internal timer interrupt is reached at every 1000 $\mathrm{Hz}$ in order to synchronize the control loop cycle. If the controller receives any command from the MLP, it sends the command to two arm motors and updates the gripper motor command. The user is capable of enabling/disabling motor torque, updating goal position and velocity, and updating control parameters including PID gains.

Since the tendons are tensioned by the gripper DC motor in the closed configuration, the gripper does not require a calibration when the controller board is power cycled. However, the tendons may stretch over time, requiring recalibration. During the calibration step, the control algorithm continues opening the gripper until it detects a hard stop (finger joint interference) using motor driver current feedback monitoring. During the normal operation, the gripper DC motor is controlled under velocity PD control at $1000 \mathrm{~Hz}$. Nominal gripper motor velocity is 50 RPM.

The arm controller board commands the 2-DOF arm at $1000 \mathrm{~Hz}$. For operator situation awareness, it sends a feedback packet to the arm driver on the MLP at $1 \mathrm{~Hz}$. This packet includes arm motor current, velocity, position, and temperature.

To enable crew to back-drive the arm, the controller compares the disturbance torque to a threshold $\alpha$. When the threshold is exceeded, it turns off the motor torque for $\beta$ seconds and signals an error message to the Astrobee's MLP. In this way, the controller provides a simple form of impedance control.

\section{EXPERIMENTAL RESULTS}

\section{A. Gripper Pull Test}

The gripper detachment force from a handrail is calibrated so that crew are unlikely to dislodge a perched Astrobee through casually bumping it, yet it is easy to intentionally remove an Astrobee that is blocking crew movement in an emergency. The ideal force range is approximately 1-5 lbf (4.45-22.2 N). We experimentally measured the detachment force with the prototype arm by pulling a perched Astrobee directly away from a wall while grasping a handrail. The mean detachment force from 5 trials was $6.43 \mathrm{~N}$, within the ideal range. We also note this value is five times greater than the Astrobee's maximum thrust capability; there is little risk of Astrobee detaching itself during its grasp check, assuming successful envelopment.

\section{B. Pan Motion on Micro-gravity Simulating Surface}

Fig. 7 shows the perching arm operating on Astrobee prototype 4 [25]. The air bearing was running such that Astrobee was free to drift on the granite table, but the propulsion system was turned off for this test of the perching arm. The test operator initially held the Astrobee robot steady 


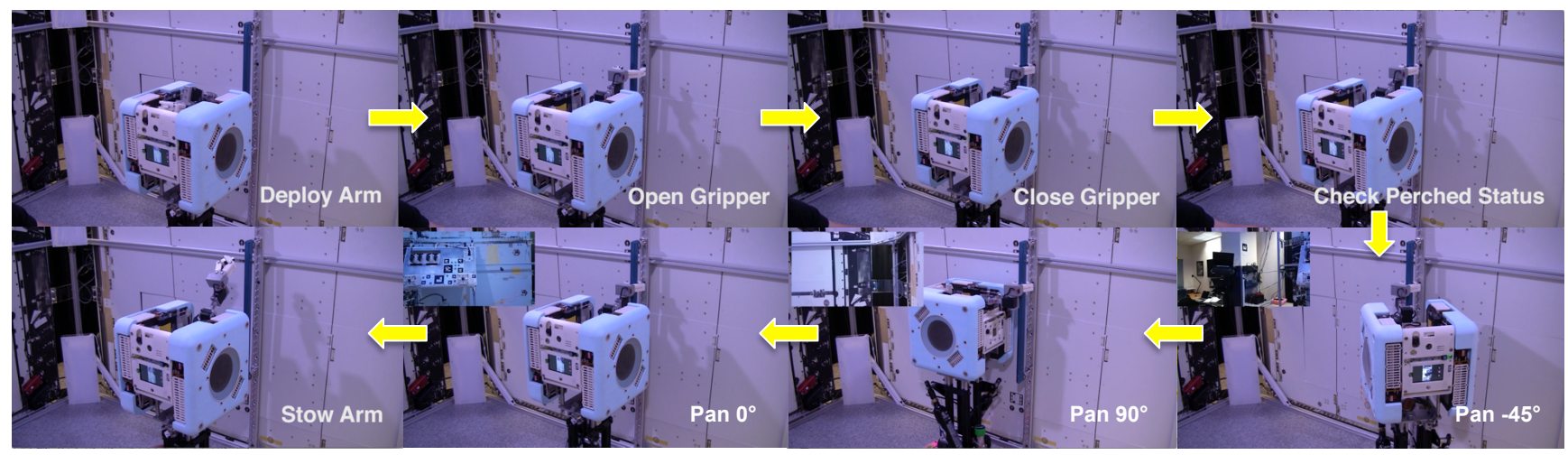

Fig. 7. Snapshot of testing the pan motion on micro-gravity simulating surface.

while the arm was commanded to deploy slowly and the gripper was commanded to open. (In flight, slow movement minimizes dynamic effects on the robot.) The test operator then manually moved the robot so the gripper contacted the handrail as it would after a successful perching approach. The gripper was commanded to close slowly, enveloping the handrail. The test operator checked for a solid grip by applying a gentle pulling force directly away from the wall, then released the robot. (In flight, both the propulsion system and compute-intensive navigation software would be turned off at this point to reduce power consumption.) The arm was then successfully commanded through a pan motion, repointing the SciCam camera attached on the opposite side of robot. SciCam images are shown as insets at the upper left.

In flight, the robot will be able to use its propulsion system to autonomously approach a handrail and perch, but that functionality is not implemented and left as future work.

\section{CONCLUSION}

This paper has presented the design and control of lightweight 3-DOF compliant perching arm for the Astrobee robot. The arm can be used for future manipulation research in zero-gravity; Astrobee robots will be able to grasp each other in flight, to simulate orbital capture scenarios. The arm also allows Astrobee to perch on handrails, so it can dwell for extended periods with reduced power consumption and disturbance to crew. The arm is small and light enough to be accommodated completely within Astrobee's payload bay, for collision safety. The under-actuated tendon-driven gripper is sized to grasp ISS handrails as well as a variety of other objects. It uses springs to close and a motorized tendon to open, so the gripper passively stays closed when powered down, and can be opened manually by the crew. We hope that the modular end-effector design will provide an opportunity to future payload developers. Co-located RGB camera and LIDAR sensors provide perception. Developing advanced torque control for the arm and autonomous perching navigation are left as a further work.

\section{ACKNOWLEDGMENT}

We would like to thank the ISS Payloads Office, the JSC Flight Operations Directorate, ISS Avionics and Software, the Advanced Exploration Systems program, the ISS SPHERES team, and the Astrobee team for their collaboration.

This research was supported by the NASA Game Changing Development Program (NASA Space Technology Mission Directorate) and the ISS SPHERES Facility (NASA Human Exploration and Operations Mission Directorate).

\section{REFERENCES}

[1] M. Bualat, J.Barlow, T. Fong, C. Provencher, T. Smith and A. Zuniga, "Astrobee: Developing a free-flying robot for the international space station," in Proc. AIAA Space 2015, Aug. 2015, pp. 1-10.

[2] J. Yoo, I.-W. Park, V. To, J. Lum and T. Smith, "Avionics and perching systems of free-flying robots for the international space station," in Proc. IEEE Int. Symp. Sys. Eng., Sept. 2015.

[3] T. Smith, J. Barlow, M. Bualat, T. Fong, C. Provencher, H. Sanchez, E. Smith and the Astrobee Team, "Astrobee: A new platform for freeflying robotics on the international space station," in Proc. Int. Symp. Artificial Int. Robot. Autom. Space, Jun. 2016.

[4] B. Coltin, J. Fusco, Z. Moratto, O. Alexandrov and R. Nakamura, "Localization from visual landmarks on a free-flying robot," in Proc. IEEE/RSJ Int. Conf. Intell. Robot. Syst., Oct. 2016, pp. 4377-4382.

[5] Evan Ackerman, IEEE Spectrum, How NASA's Astrobee robot is bringing useful autonomy to the ISS, Available: http://spectrum.iee.org/automaton/robotics/space-robots/how-nasaastrobee-robot-is-bringing-useful-autonomy-to-the-iss

[6] J. L. Broyan, et al. "Logistics reduction technologies for exploration missions," in Proc. AIAA Space, 2014.

[7] M. Kovac, J. Germann, C. Hurzeler, R. Y. Siegwart and D. Floreano, "A perching mechanism for micro aerial vehicles," J. Micro-Nano Mech, vol. 5, pp. 77-91, 2009.

[8] E. Brown, N. Rodenberg, J. Amend, A. Mozeika, E. Stelz, M. R. Zakin, H. Lipson and H. M. Jaeger, "Universal robotic gripper based on the jamming of granular material," in Proc. Nat. Academy Sci., Nov. 2010, pp. 18809-18814.

[9] J. Amend, E. Brown, N. Rodenberg, H. J. Jaeger and H. Lipson, "A positive pressure universal gripper based on the jamming of granular material," IEEE Trans. Robot., vol. 28, no. 2, pp. 341-350, 2012.

[10] H. Prahlad, R. Pelrine, S. Stanford, J. Marlow and R. Kornbluh, "Electroadhesive robotis - Wall climbing robots enabled by a novel, robust, and electrically controllable adhesion technology," in Proc. IEEE Int. Conf. Robot. Autom., May 2008, pp. 3028-3033.

[11] D. Mellinger, Q. Linsey, M. Shomin and V. Kumar, "Design, modeling, estimation and control for aerial grasping and manipulation," in Proc. IEEE/RSJ Int. Conf. Intell. Robot. Syst., Sep. 2011, pp. 2668-2673.

[12] A. Desbiens, A. Asbeck and M. Cutkosky, "Landing, perching and taking off from vertical surfaces," Int. J. Robot. Res., vol. 30, no. 5, pp. 355-370, 2011. 


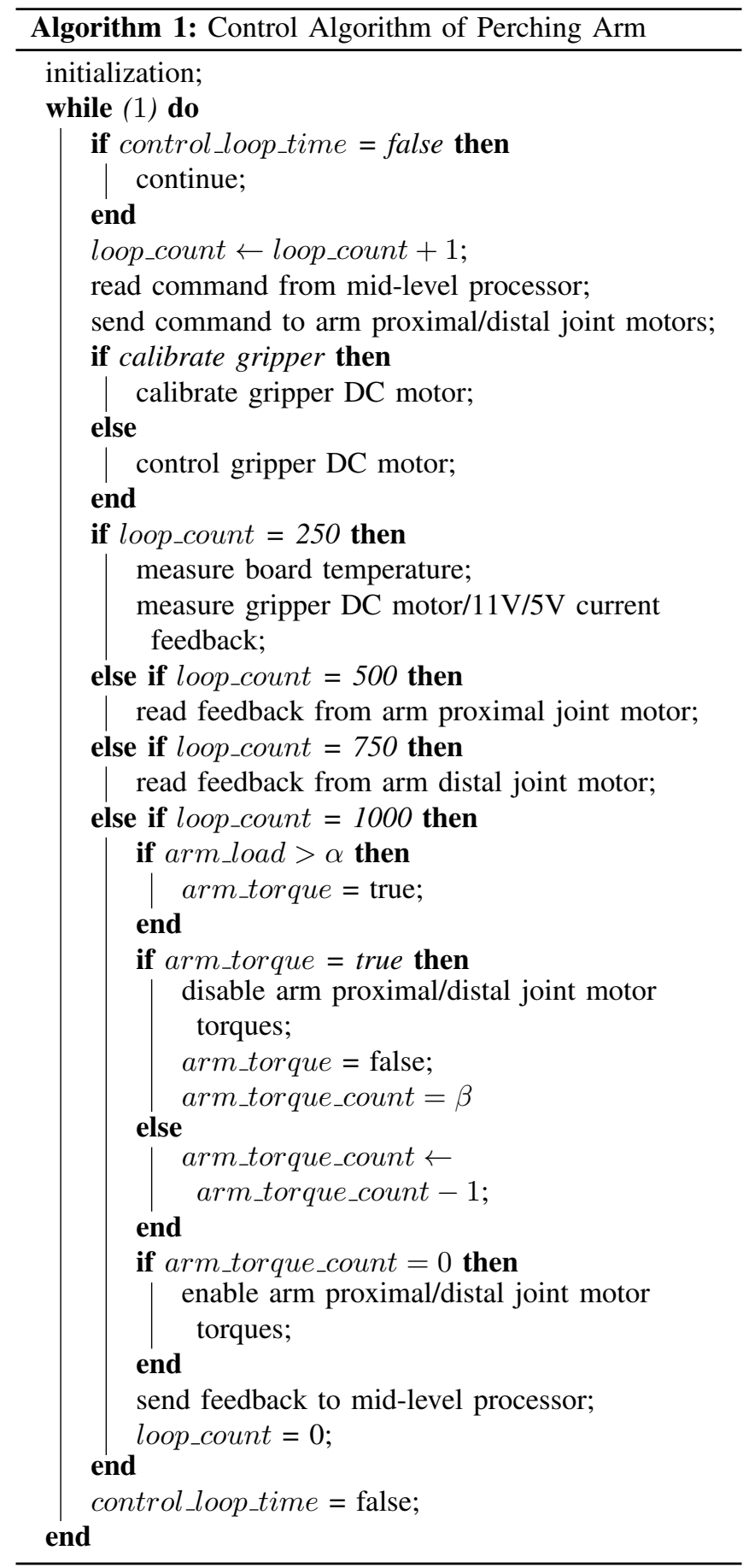

[13] A. Parness, M. Frost, N. Thatte and J. King, "Gravity-independent mobility and drilling on natural rock using microspines," in Proc. IEEE Int. Conf. Robot. Autom., May 2012, pp. 3437-3442.

[14] L. Daler, A. Klaptocz, A. Briod, M. Setti and D. Floreano, "A perching mechanism for flying robots using a fibre-based adhesive," in Proc. IEEE Int. Conf. Robot. Autom., May 2013, pp. 4418-4423.

[15] E. Hawkes, D. Christensen, E. Eason, M. Estrada, M. Heverly, E. Hilgemann, H. Jiang, M. Pope, A. Parness and M. Cutkosky, "Dynamic surface grasping with directional adhesion," in Proc. IEEE/RSJ Int. Conf. Intell. Robot. Syst., Nov. 2013, pp. 5487-5493.

[16] M. Estrada, E. Hawkes, D. Christensen and M. Cutkosky, "Perching and vertical climbing: Design of a multimodal robot," in Proc. IEEE Int. Conf. Robot. Autom., May 2014, 4215-4221.

[17] N. Wettels and A. Parness, "Advances in fibrillar on-off polymer adhesive: sensing and engagement speed," in Proc. IEEE/RSJ Int. Conf. Intell. Robot. Syst., Sep. 2014, pp. 4266-4271.

[18] L. Odhner, R. Ma and A. Dollar, "Precision grasping and manipulation of small objects from flat surfaces using underactuated fingers," in Proc. IEEE Int. Conf. Robot. Autom., May 2012, pp. 2830-2835.

[19] C. Doyle, J. Bird, T. Isom, J. Kallman, D. Bareiss, D. Dunlop, R. King, J. Abbott and M. Minor, "An avian-inspired passive mechanism for quadrotor perching," IEEE/ASME Trans. Mechatronics, vol. 18, no. 2 , pp. 506-517, 2013.

[20] W. Chi, K. H. Low, K. H. Hoon and J. Tang, "An optimized perching mechanism for autonomous perching with a quadrotor," in Proc. IEEE Int. Conf. Robot. Autom., May 2014, pp. 3109-3115.

[21] M. Ciocarlie, F. M. Hicks, R. Holmberg, J. Hawke, M. Schlicht, J. Gee, S. Stanford and R. Bahadur, "The Velo gripper: A versatile singleactuator design for enveloping, parallel and fingertip grasps," Int. $J$. Robot. Res., vol. 33, no. 5, pp. 753-767, 2014.

[22] A. M. Dollar and R. D. Howe, "The highly adaptive SDM hand Design and performance evaluation," Int. J. Robot. Res., vol. 29, no. 5, pp. 585-597, 2010.

[23] Robotic Manipulation and Mobility Lab, Columbia University, Perching gripper design, Available: http://roam.me.columbia.edu/perchinggripper-design

[24] Ken Caluwaerts, dsPIC33E CAN and UART bootloader, Available: https://github.com/kcaluwae/dspic33e-bootloader

[25] In-Won Park, Astrobee P4C Perching Arm Pan on Micro-Gravity Table, Available: https://youtu.be/RGVbAOrdh2M 